\title{
Nonlinear Elasticity in Wurtzite GaN/AlN Planar Superlattices and Quantum Dots
}

\author{
S.P. ŁePKOWSKI ${ }^{a, *}$, J.A. MAJeWski ${ }^{b}$ AND G. JURCZAK ${ }^{c}$
}

${ }^{a}$ Unipress, Institute of High Pressure Physics, Polish Academy of Sciences

Sokołowska 29, 01-142 Warszawa, Poland

${ }^{b}$ Institute of Theoretical Physics, Warsaw University

Hoża 69, 00-681 Warszawa, Poland

${ }^{c}$ Institute of Fundamental Technological Research

Świȩtokrzyska 21, 00-049 Warszawa, Poland

The elastic stiffness tensors for wurtzite GaN and AlN show a significant hydrostatic pressure dependence, which is the evidence of nonlinear elasticity of these compounds. We have examined how pressure dependence of elastic constants for wurtzite nitrides influences elastic and piezoelectric properties of GaN/AlN planar superlattices and quantum dots. Particularly, we show that built-in hydrostatic pressure, present in both quantum wells of the GaN/AlN superlattices and GaN/AlN quantum dots, increases significantly by $0.3-0.7 \mathrm{GPa}$ when nonlinear elasticity is used. Consequently, the compressive volumetric strain in quantum wells and quantum dots decreases in comparison to the case of the linear elastic theory. However, the $z$-component of the built-in electric field in the quantum wells and quantum dots increases considerably when nonlinear elasticity is taken into account. Both effects, i.e., a decrease in the compressive volumetric strain as well as an increase in the built-in electric field, decrease the band-to-band transition energies in the quantum wells and quantum dots.

PACS numbers: 78.67.- $-\mathrm{n}, 62.20 . \mathrm{Dc}, 62.50 .+\mathrm{p}$

\section{Introduction}

The strain effects in semiconductor nanostructures, i.e. quantum wells (QWs), wires or dots (QDs), are usually described using the standard elasticity theory with elastic constants independent on the strain (so-called linear theory). However, the influence of nonlinear elastic properties of semiconductors on elec-

*corresponding author; e-mail: slawek@unipress.waw.pl 
tronic and optical properties of nanostructures has recently attracted significant attention. First, Frogley et al. proposed that a pressure dependence of elastic constants in GaAs and InAs is required to explain an anomalously small pressure coefficient of band gap $\left(\mathrm{d} E_{\mathrm{G}} / \mathrm{d} P\right)$ in strained InGaAs layers [1]. Second, Ellaway et al. calculated pressure dependences of elastic constants for InAs and discussed their influence on the properties of InAs/GaAs QDs [2,3]. They noticed that a hydrostatic strain component in the InAs/GaAs QDs is significantly overestimated by calculations based on linear theory of elasticity. We have recently calculated the pressure dependence of elastic stiffness tensor for zinc-blende InN, GaN, and AlN and showed that the nonlinear elasticity in zinc-blende GaN and $\mathrm{InN}$ is crucial for determination of $\mathrm{d} E_{\mathrm{G}} / \mathrm{d} P$ in cubic InGaN/GaN QWs $[4,5]$.

In this work, we examine how nonlinear elasticity of wurtzite GaN influences elastic and piezoelectric properties of GaN/AlN planar superlattices (SLs) and quantum dots. Performing ab initio calculations in the framework of plane-wave pseudopotential implementation of the density-functional theory [6], we have found the following pressure dependence of elastic constants for wurtzite GaN (in GPa):

$$
\begin{aligned}
& C_{11}=322+5.23 P-0.033 P^{2}, \\
& C_{12}=112+3.90 P-0.018 P^{2}, \\
& C_{13}=79+3.27 P-0.001 P^{2}, \\
& C_{33}=354+6.7 P-0.059 P^{2}, \\
& C_{44}=83+1.33 P-0.023 P^{2},
\end{aligned}
$$

where $P$ is the hydrostatic pressure. In GaN/AlN SLs and QDs, the GaN region is subjected to compressive stress which possesses the non-zero hydrostatic component, the so-called built-in hydrostatic pressure. It is usually defined as

$$
P=-\operatorname{Tr}\left(\sigma_{\alpha, \beta}\right) / 3,
$$

where $\sigma_{\alpha, \beta}$ is the Cauchy stress tensor. In the sections to come, we show how the pressure dependent elastic constants of wurtzite GaN, given by Eqs.(1) and (2), influence elastic and piezoelectric properties of GaN/AlN SLs and QDs.

\section{GaN/AlN planar superlattices}

In planar SLs, the strain tensor is described by the following diagonal elements [7]:

$$
\begin{aligned}
\varepsilon_{x x, i} & =\frac{a_{\mathrm{s}}-a_{0, i}}{a_{0, i}}, \\
\varepsilon_{z z, i} & =-\frac{2 C_{13}(P)}{C_{33}(P)} \varepsilon_{x x, i}, \quad i=\mathrm{qw}, \mathrm{br},
\end{aligned}
$$

where $i$ indicates quantum wells and barriers, $a_{0, i}$ is the equilibrium lattice constant of QWs or barriers, respectively. The lateral constant of the whole super- 
lattice, $a_{\mathrm{s}}$, takes the value that minimizes the total elastic energy. To determine $a_{\mathrm{s}}$, we consider two cases: (i) SLs strained to thick buffer of AlN, which gives $a_{\mathrm{S}}=a_{0, \mathrm{br}}$ and (ii) free standing SLs, for which $a_{\mathrm{S}}$ is obtained by minimizing the total elastic energy in the SLs [7]:

$$
a_{\mathrm{s}}=\frac{a_{0, \mathrm{qw}} L_{\mathrm{qw}} G_{\mathrm{qw}}+a_{0, \mathrm{br}} L_{\mathrm{br}} G_{\mathrm{br}}}{L_{\mathrm{qw}} G_{\mathrm{qw}}+L_{\mathrm{br}} G_{\mathrm{br}}},
$$

where: $G_{i}=2\left[C_{11, i}(P)+C_{12, i}(P)-2\left(C_{13, i}(P)\right)^{2} / C_{33, i}(P)\right], i=\mathrm{qw}$, br and $L_{\mathrm{qw}(\mathrm{br})}$ is the thickness of QWs or barriers, respectively. The built-in hydrostatic pressure in GaN QWs, defined by Eq. (2), can be determined from the Hooke's law:

$$
P=-\frac{2}{3}\left[C_{11}(P)+C_{12}(P)-2 \frac{\left(C_{13}(P)\right)^{2}}{C_{33}(P)}\right] \varepsilon_{x x, \mathrm{qw}} .
$$

To determine the built-in electric field in the QWs, we consider first spontaneous and piezoelectric polarization. The non-vanishing $z$-components (in the [0001] direction, along $c$-axis) of the total piezoelectric polarization are given by

$$
P_{\mathrm{tot}, i}=2 e_{31, i} \varepsilon_{x x, i}+e_{33, i} \varepsilon_{z z, i}+P_{\mathrm{spon}, i}, \quad i=\mathrm{qw}, \mathrm{br},
$$

where $e_{31, i}$ and $e_{33, i}$ are elements of the piezoelectric tensor and $P_{\mathrm{spon}, i}$ is the spontaneous polarization. Then, the $z$-component of the electric field vector in the QWs of the superlattice is the following [8]:

$$
E_{\mathrm{qw}}=\left(P_{\mathrm{tot}, \mathrm{br}}-P_{\mathrm{tot}, \mathrm{qw}}\right) \frac{L_{\mathrm{br}}}{L_{\mathrm{qw}} \chi_{\mathrm{br}}+L_{\mathrm{br}} \chi_{\mathrm{qw}}},
$$

where $\chi_{\mathrm{qw}(\mathrm{br})}$ is the static dielectric constant for the QWs and barriers, respectively.

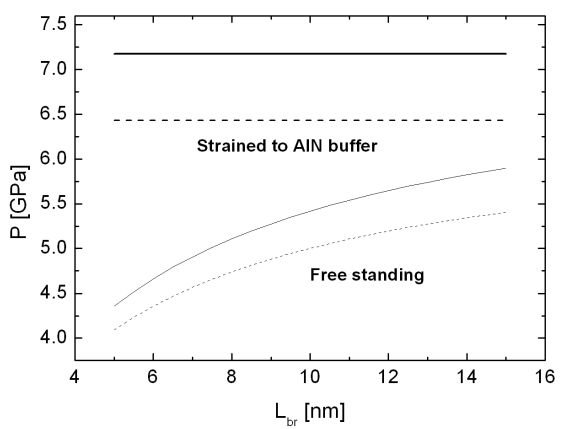

Fig. 1. The built-in hydrostatic pressure calculated for free standing and strained to the AlN buffer GaN/AlN superlattices with $L_{\mathrm{qw}}=3 \mathrm{~nm}$ and $L_{\mathrm{br}}$ varying from 5 to $15 \mathrm{~nm}$. The solid lines indicate results obtained with the nonlinear elasticity, whereas the dash lines correspond to the linear elasticity. Note that for the SLs strained to the AlN buffer the independence of the built-in hydrostatic pressure on $L_{\mathrm{br}}$ results from the constant value of $a_{\mathrm{s}}$. 
In Fig. 1 we present the built-in hydrostatic pressure calculated for GaN/AlN SLs with $L_{\mathrm{qw}}=3 \mathrm{~nm}$ and $L_{\mathrm{br}}$ varying from 5 to $15 \mathrm{~nm}$, where the results for free standing SLs as well as SLs strained to thick AlN buffer are shown. One can see that the usage of nonlinear elastic theory results in a significant increase in $P$ by 0.3-0.7 GPa, as compared to the case of linear elasticity. In Fig. 2 we show the volumetric strain, $\varepsilon_{\mathrm{vol}}=\operatorname{Tr}\left(\varepsilon_{\alpha, \beta}\right)$, calculated for GaN/AIN SLs with $L_{\mathrm{qw}}=3 \mathrm{~nm}$
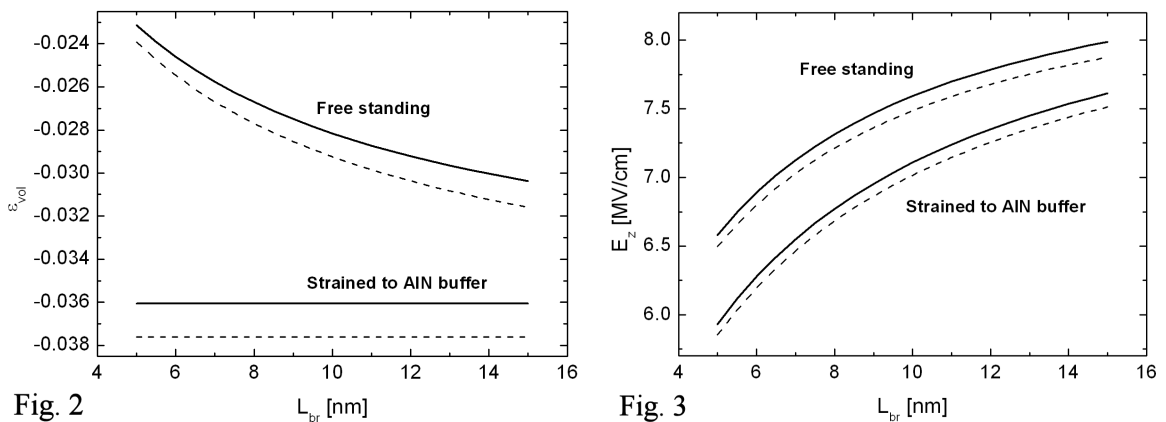

Fig. 2. The volumetric strain calculated for free standing and strained to the AlN buffer GaN/AlN superlattices with $L_{\mathrm{qw}}=3 \mathrm{~nm}$ and $L_{\mathrm{br}}$ varying from 5 to $15 \mathrm{~nm}$. The solid lines indicate results obtained with the nonlinear elasticity, whereas the dash lines correspond to the linear elasticity. Note that for the SLs strained to the AlN buffer the independence of the volumetric strain on $L_{\mathrm{br}}$ results from the constant value of $a_{\mathrm{s}}$.

Fig. 3. The built-in electric field calculated for free standing and strained to the AlN buffer GaN/AlN superlattices with $L_{\mathrm{qw}}=3 \mathrm{~nm}$ and $L_{\mathrm{br}}$ varying from 5 to $15 \mathrm{~nm}$. The solid lines indicate results obtained with the nonlinear elasticity, whereas the dash lines correspond to the linear elasticity.

and $L_{\mathrm{br}}$ changing from 5 to $15 \mathrm{~nm}$. The absolute value of $\varepsilon_{\mathrm{vol}}$ is smaller when nonlinear elasticity is used. The built-in electric field in the QWs is shown in Fig. 3. One can see that $E_{\mathrm{qw}}$ increases considerably by $0.08-0.13 \mathrm{MV} / \mathrm{cm}$ when nonlinear elasticity is taken into account.

\section{GaN/AlN quantum dots}

In this section, we analyze the influence of the nonlinear elasticity on elastic, piezoelectric and optical properties on wurtzite GaN/AlN QDs. We have developed a numerical model, based fully on the finite element method, which determines strain distribution, piezoelectric fields, and electronic states in wurtzite QDs [9]. Our model consists of three parts. In the first part, we solve the boundary-value problem obtained from the integration of the equilibrium equation for the Cauchy stress tensor, $\operatorname{div} \boldsymbol{\sigma}=0$. At the beginning, we determine the initial strain and stress fields for the stiffness tensor at zero pressure $C_{\alpha \beta}(0)$. Then we calculate $P$ using Eq. (2) and from Eq. (1) we obtain modified elastic constants $C_{\alpha \beta}(P)$ 
corresponding to this pressure. The elastic constants calculated in such a way are then used to obtain new strain and stress distributions. This iteration procedure is repeated until convergent solution is obtained. In the second step, we calculate piezoelectric polarization field and solve the Poisson equation to obtain the distribution of electrostatic potential. Finally, in the third step, we solve $8 \times 8 \mathrm{k} \cdot \mathrm{p}$ Hamiltonian of the wurtzite quantum dots to obtain electronic energy levels in the dot.

We have performed calculation for a single GaN/AIN QD having the shape of truncated hexagonal pyramid placed on $0.5 \mathrm{~nm}$ thick wetting layer. The height of the QD was $3 \mathrm{~nm}$, the base diameter was $26.5 \mathrm{~nm}$, and the angle between the base of the dot and a side wall was $\pi / 6$. In Fig. 4 we present profiles of the built-in hydrostatic pressure taken from the center of the QDs, along $z$ axis [0001], parallel to the direction of growth. One can see that the usage of the nonlinear elastic theory results in a significant increase in $P$ in the QD region by about 0.4 GPa. In Fig. 5 we show the profiles of volumetric strain, $\varepsilon_{\mathrm{vol}}=\operatorname{Tr}(\varepsilon)$, taken also along $z$ axis. The magnitude of $\varepsilon_{\mathrm{vol}}$ in the QD region decreases by $6.5 \%$ when nonlinear elasticity is used. Interestingly, the average $z$-component of the built-in electric field in the QD increases considerably by $0.15 \mathrm{MV} / \mathrm{cm}$ when nonlinear elasticity is taken into account. Both effects, i.e., a decrease in volumetric strain as well as an increase in the built-in electric field, decrease the band-to-band transition energies in the QDs. Indeed, we observe that the fundamental transition band-to-band energy decreases by about $60 \mathrm{meV}$, compared to the case when the linear elastic theory is used.
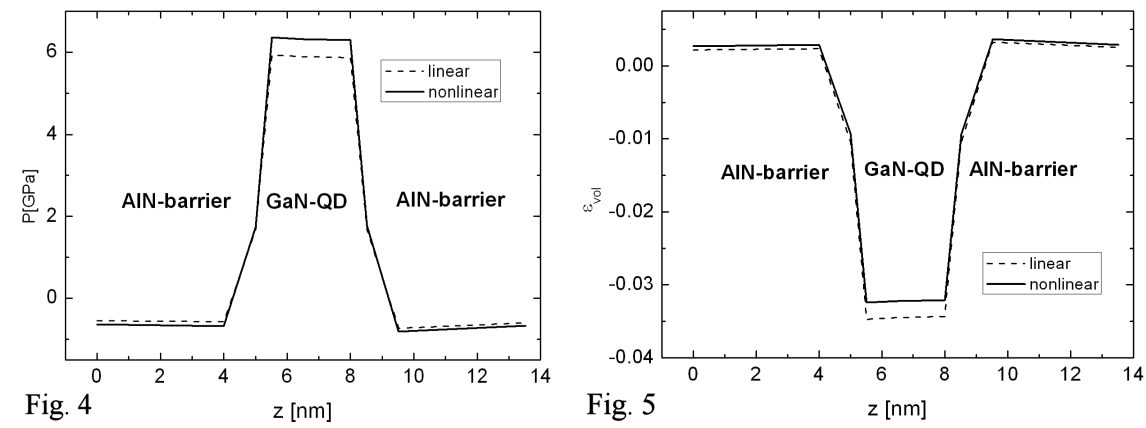

Fig. 4. The built-in hydrostatic pressure calculated for wurtzite GaN/AlN quantum dot having the shape of truncated hexagonal pyramid (the height of the QD was $3 \mathrm{~nm}$, the base diameter was $26.5 \mathrm{~nm}$ ). The solid line indicates results obtained with the nonlinear elasticity, whereas the dash line corresponds to the linear elasticity.

Fig. 5. The volumetric strain calculated for wurtzite GaN/AlN quantum dot having the shape of truncated hexagonal pyramid (the height of the QD was $3 \mathrm{~nm}$, the base diameter was $26.5 \mathrm{~nm}$ ). The solid line indicates results obtained with the nonlinear elasticity, whereas the dash line corresponds to the linear elasticity. 


\section{Conclusions}

In summary, we have studied the effect of nonlinear elastic properties of wurtzite nitrides on elastic and piezoelectric properties of GaN/AIN SLs and QDs. Our studies reveal that the built-in hydrostatic pressure, volumetric strain, and polarization induced electric field are influenced by the nonlinear elastic effects in the same manner, in both, SLs and QDs. For the GaN/AlN SLs we have observed that the usage of the nonlinear elasticity results in the significant increase in the built-in hydrostatic pressure in the GaN QWs by $0.3-0.7 \mathrm{GPa}$. Consequently, the compressive volumetric strain in GaN QWs decreases compared to the case of the linear elastic theory. However, the $z$-component of the built-in electric field in the QWs increases considerably by $0.08-0.13 \mathrm{MV} / \mathrm{cm}$ when nonlinear elasticity is taken into account. Qualitatively, the same effects have been observed for GaN/AlN QDs. For the typical GaN/AlN QD having the height of $3 \mathrm{~nm}$ and the base diameter of $26.5 \mathrm{~nm}$, we have shown that the usage of the nonlinear elasticity leads to the increase in $P$ in the QD region by about $0.4 \mathrm{GPa}$ and the relative decrease in $\varepsilon_{\mathrm{vol}}$ by $6.5 \%$ as compared to the case of the linear elasticity. The average $z$-component of the built-in electric field in the QD region increases considerably by $0.15 \mathrm{MV} / \mathrm{cm}$. Both effects, i.e., the decrease in volumetric strain as well as the increase in the built-in electric field, result in the significant decrease in the fundamental transition band-to-band energy by about $60 \mathrm{meV}$.

\section{Acknowledgments}

This work has been supported by the State Committee for Scientific Research (Poland) Project No. 4T11F 00825.

\section{References}

[1] M.D. Frogley, J.R. Downes, D.J. Dunstan, Phys. Rev. B 62, 13612 (2000).

[2] S.W. Ellaway, D.A. Faux, Phys. Status Solidi B 235, 437 (2003).

[3] S.W. Ellaway, D.A. Faux, J. Appl. Phys. 92, 3027 (2002).

[4] S.P. Łepkowski, J.A. Majewski, Solid State Commun. 131, 763 (2004).

[5] S.P. Łepkowski, J.A. Majewski, Acta Phys. Pol. A 105, 559 (2004).

[6] G. Kresse, J. Furthmuller, Phys. Rev. B 54, 11169 (1996).

[7] E. Anastassakis, Phys. Rev. B 46, 13244 (1992).

[8] F. Bernardini, V. Fiorentini, Phys. Status Solidi B 216, 391 (1999).

[9] S.P. Łepkowski, G. Jurczak, P. Dłużewski, T. Suski, in: GaN, AlN, InN and their Alloys, Eds. Ch. Wetzel, B. Gil, M. Kuzuhara, M. Manfra, Vol. 831, Mater Res. Soc. Symp. Proc., Warrendale (PA) 2005, p. E11.14. 